ORIGINAL

\title{
Assay of serum E2 concentration in postmenopausal breast cancer patients using a high-sensitivity RIA method is generally useful
}

\author{
Masami Morimoto ${ }^{1}$, Masako Takahashi ${ }^{2}$, Junko Honda ${ }^{3}$, Takahiro Yoshida ${ }^{1}$, Mitsuteru Yoshida ${ }^{1}$, Hiroaki Toba ${ }^{1}$, \\ Issei Imoto ${ }^{4}$, Akira Tangoku' ${ }^{1}$, and Mitsunori Sasa ${ }^{2}$

\begin{abstract}
${ }^{1}$ Department of Oncological and Regenerative Surgery, Institute of Health Biosciences, the University of Tokushima, Tokushima, Japan, ${ }^{2}$ Department of Surgery, Tokushima Breast Care Clinic, Tokushima, Japan, ${ }^{3}$ Department of Surgery, Higashitokushima National Hospital, Tokushima, Japan, ${ }^{4}$ Department of Human Genetics, Institute of Health Biosciences, the University of Tokushima Graduate School, Tokushima, Japan
\end{abstract}

\begin{abstract}
Background : Serum E2 must be monitored for aromatase inhibitor (AI) therapy, but conventional assays lack sensitivity. Subjects/Methods : Forty amenorrheic breast cancer patients scheduled for AI treatment but requiring hormonological confirmation of their menopausal status were studied. Serum E2 data generated by high-sensitivity RIA and by LC-MS/MS were analyzed for correlation. Results : RIA gave a higher E2 value than LC-MS/MS in $62 \%$ of cases, but there was a significant positive correlation. Patients whose E2 levels by RIA were $\geq 2.5 \mathrm{pg} / \mathrm{mL}$ higher than those by LC-MS/MS (RIA-H group) and all other patients (RIA-N group) were compared. Both groups showed strong correlations between the two assay methods. With both methods patients with a high BMI had significantly elevated E2. Multiple regression analysis used age, age at menarche, number of births and BMI as explanatory variables. Significant variables were the BMI with LC-MS/MS, and both BMI and age with RIA. The RIA-H and RIA-N groups showed no difference in regard to the BMI, whereas the age was significantly lower in the RIA-H group. Summary : Serum E2 levels determined for postmenopausal women by RIA and LC-MS/MS generally correlated well. High-sensitivity RIA is a potentially useful clinical assay, but it overestimated serum E2 in some women. J. Med. Invest. 63 : 236 -240, August, 2016
\end{abstract}

Keywords : Breast cancer, post-menopausal women, serum E2, high-sensitivity RIA, LC-MS/MS

\section{BACKGROUND}

Approximately $80 \%$ of female breast cancer patients are hormonereceptor-positive, and aromatase inhibitors (AI) are widely used to treat breast cancer in postmenopausal women $(1,2)$. AI drugs express their anti-tumor effects by reducing the levels of estradiol (E2) in the blood and tumors by about $90 \%(3,4)$. However, it has been reported that serum $\mathrm{E} 2$ does not decrease in some patients, thus necessitating monitoring of $\mathrm{E} 2$ in the early stage of AI therapy (5-8).

The serum level of $\mathrm{E} 2$ in postmenopausal women is defined as being $10 \sim 20 \mathrm{pg} / \mathrm{mL}$ or less, but the lower limit of detection of most E2 assay kits that are used in clinics in Japan is about $20 \mathrm{pg} / \mathrm{mL}$ (9). For that reason, it is difficult to accurately determine and monitor the serum E2 level in many postmenopausal women with those commonly used assay systems. Serum E2 levels have also been measured by a high-sensitivity radio-immunoassay (RIA) method and by a high-sensitivity ELISA method $(8,10)$, but it is anticipated that cross-reactions and non-specific reactions will occur with those methods. In particular, it is thought that, in the case of measuring trace levels of serum E2, these unfavorable reactions will affect the credibility of the measured values. One report, from Sweden, measured the serum E2 levels in postmenopausal women with a high-sensitivity RIA method and with ELISA and then

Received for publication March 18, 2016 ; accepted May 8, 2016.

Address correspondence and reprint requests to Masami Morimoto, Department of Oncological and Regenerative Surgery, Institute of Health Biosciences, the University of Tokushima, 3-18-15, Kuramoto-Cho, Tokushima 770-8509, Japan and Fax : +81-88-633-7144. compared the data with those determined by a liquid chromatography-tandem mass spectrometry (LC-MS/MS) method (8). On the other hand, obesity was reported to affect the serum E2 level in postmenopausal women (10). Postmenopausal Japanese women are said to be less obese than postmenopausal women in the West, which raises the possibility that the serum E2 results may differ in those two populations. Accordingly, we assayed the serum E2 levels in postmenopausal Japanese women with breast cancer by a high-sensitivity RIA method and by an LC-MS/MS method and compared those results.

\section{MATERIALS AND METHODS}

The study cohort consisted of 40 female breast cancer patients who were being treated at Tokushima Breast Care Clinic from June through December of 2013 and required hormonological confirmation of their menopausal status to clear the way for starting AI therapy. The analyzed clinical factors were age, age at menarche, number of births, body mass index (BMI), clinical stage, ER (estrogen receptor), PgR (progesterone receptor) and HER2 (human epidermal growth factor receptor type2). The mean age of the patients was $51.5 \pm 6.3$ years. As prior treatments, 12 patients had been administered neoadjuvant chemotherapy, 15 had been administered post-operative chemotherapy and 16 patients had received hormonal therapy, whereas 8 patients were naïve (Table 1 ).

Prior to being administered an AI drug, the serum E2 levels of all the patients were assayed by high-sensitivity RIA and by LC-MS/ MS. The high-sensitivity RIA measurements were subcontracted to FALCO Biosystems Ltd. (Kyoto, Japan), while the LC-MS/MS 
Table 1. Clinical Factors

\begin{tabular}{ll}
\hline Age & $51.5 \pm 6.3(37-71)$ \\
BM1* & $24.1 \pm 5.0(17.9-37.2)$ \\
Age at Menarche & $12.3 \pm 1.8(10-15)$ \\
Number of Birth & $1.9 \pm 0.6(0-3)$ \\
Stage & 0, I, IIA, IIB, III, IV : $2,19,8,9,1,1$ cases \\
ER & Positive, Negative $: 40,0$ cases \\
PgR & Positive, Negative $: 33,7$ cases \\
HER2 & Positive, Negative $: 11,29$ cases \\
Prior-treatment & Neo-adjuvant chemotherapy $: 12$ cases \\
& Post-operative adjuvant chemotherapy : 15 cases \\
& Adjuvant hormone therapy $: 16$ cases \\
& No : 8 cases \\
\hline
\end{tabular}

*Body mass index

measurements were performed by ASKA Pharmaceutical Medical Co., Ltd. (Kanagawa, Japan). The patients' blood samples were collected by FALCO Biosystems Ltd., and the sera were separated and divided into 2 aliquots each. One of the aliquots was subjected to high-sensitivity RIA, and the other to LC-MS/MS. The antibody used for the high-sensitivity RIA measurements was RIACOAT. ESTRADIOL-US (SCETI MEDICAL LABO, Tokyo, Japan) (lower limit of measurement : $1.36 \mathrm{pg} / \mathrm{ml}$ ), which has the highest sensitivity of all in vitro diagnostic agents that have been approved in Japan. The intra-assay coefficient of variability (C.V.) for the highsensitivity RIA is $\leq 15 \%$. The inter-assay reproducibility C.V. is 5.8 $17.6 \%$. The rates of cross-reactions with the antibody are $100 \%$ for estradiol-17ß, $0.97 \%$ for estrone, $0.44 \%$ for estriol, $8.9 \%$ for equilenin, $1.4 \%$ for hinylestradiol, $1.1 \%$ for equilin, $<0.05 \%$ for progesterone and $<0.001 \%$ for testosterone. The intra-assay C.V. for LC-MS/ MS is $1.49 \sim 13.0 \%$ (however, for the lower limit of measurement : $18.2 \%)$. The inter-assay reproducibility C.V. is $0.4-11.2 \%$.

This study was carried out after approval had been obtained from the Ethics Committee of the University of Tokushima Graduate School (Registration No : 803).

Each of the studied patients was given a full explanation of the nature of this study and granted informed consent in writing.

\section{Statistics}

All statistical analyses were performed using R package (Ver. 3.0.2). Pearson's product-moment correlation coefficient ( $r$ ) was used to study the relationship between the E2 concentrations determined with the two assay methods and the relationship between those concentrations and the BMI. Single regression analyses were performed for the E2 concentrations determined with the two assay methods. We explored model selection using the Akaike Information Criterion (AIC) in the multivariate regression setting. We compared continuous variables between two categories using the Mann-Whitney U test. All $\mathrm{p}$ values are two-sided ; $\mathrm{p}$ values under 0.05 are considered to be statistically significant.

\section{RESULTS}

The sera from 3 of the 40 patients showed E2 levels of $\geq 10 \mathrm{pg} /$ $\mathrm{mL}$ in both the high-sensitivity RIA and LC-MS/MS assays. We analyzed the results for the other 37 patients. In $23(62 \%)$ of those patients, the E2 level was higher with the high-sensitivity RIA test than with the LC-MS/MS assay, and the two sets of assay data showed a significant but weak positive correlation $(r=0.387, p=$ 0.0179 ) (Figure. 1). The cases were stratified into two groups : a group in which the E2 level by high-sensitivity RIA was $\geq 2.5 \mathrm{pg} / \mathrm{mL}$ higher than the $\mathrm{E} 2$ value by LC-MS/MS (RIA-H group), and a

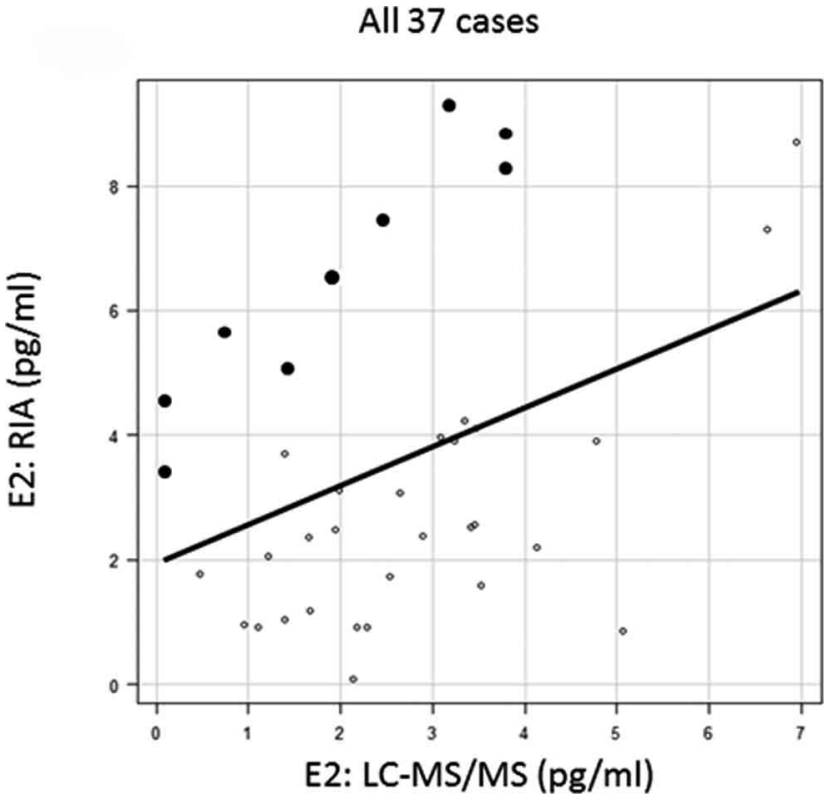

Figure 1 : Comparison of E2 values determined by high-sensitivity RIA and by LC-MS/MS ( $\mathrm{n}=37)$

High-sensitivity RIA gave a higher E2 value than LC-MS/MS in $62 \%$ of the cases, but the results with the two methods showed a significant, weakly-positive correlation $(r=0.387, p=0.0179, y=0.628 x+1.977)$. The cases were stratified into two groups : a group in which the E2 level by high-sensitivity RIA was $\geq 2.5 \mathrm{pg} / \mathrm{mL}$ higher than the E2 value by LCMS/MS (RIA-H group ; black circles), and a group comprised of all other cases (RIA-N group; white circles).

group comprised of all other cases (RIA-N group) (Figure. 1). Analysis showed significant, strong correlations between the two assay methods in both patient groups : $r=0.945, p=0.00012$ for the RIA-H group and $\mathrm{r}=0.705, \mathrm{p}=0.00003$ for the RIA-N group (Figure. 2a, 2b).

Next, the BMI and E2 level were analyzed for a correlation, and the results showed that, for the E2 data generated by both the RIA and LC-MS/MS assay methods, patients with a high BMI had a significantly higher E2 level. There was also a significant correlation between the results with the two assay methods (Figure. 3a, 3b). Multiple regression analysis was performed using the age, age at menarche, number of births and BMI as explanatory variables, and by using the AIC for variable selection. With LC-MS/MS the BMI was the only significant variable selected $(\mathrm{E} 2=0.171 \times \mathrm{BMI}-1.558$, $\mathrm{p}=0.00037)$, while with high-sensitivity RIA both the BMI ( $\mathrm{p}=$ $0.00003)$ and age $(p=0.00272)$ were significant variables $(E 2=$ 


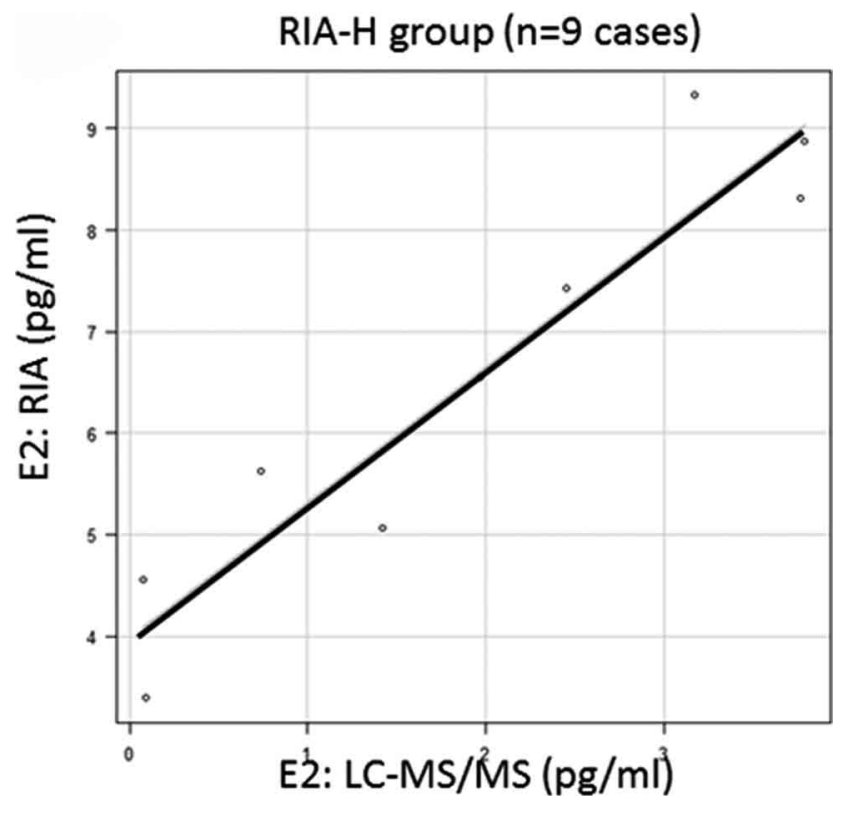

Figure 2a : Comparison of E2 values determined by high-sensitivity RIA and by LC-MS/MS for the RIA-H group $(n=9)$

The E2 values determined by both methods showed a significant, stronglypositive correlation $(\mathrm{r}=0.945, \mathrm{p}=0.00012, \mathrm{y}=1.332 \mathrm{x}+3.982)$.

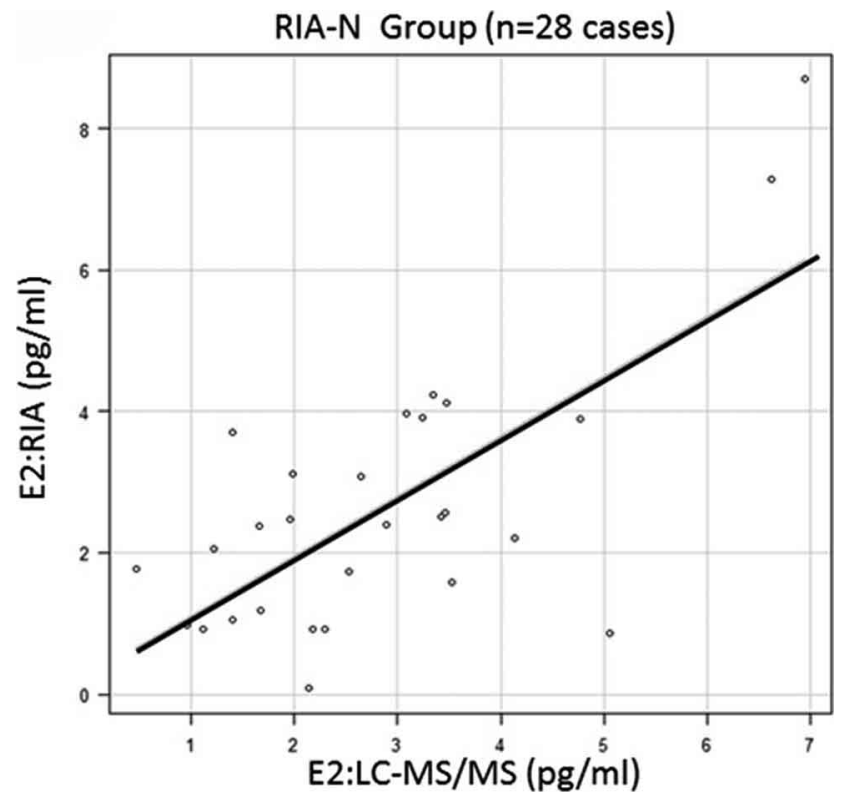

Figure $2 \mathrm{~b}$ : Comparison of $\mathrm{E} 2$ values determined by high-sensitivity RIA and by LC-MS/MS for the RIA-N group $(\mathrm{n}=28)$

The E2 values determined by both methods showed a significant, stronglypositive correlation $(r=0.705, p=0.00003, y=0.848 x+0.255)$.

$0.288 \times \mathrm{BMI}-0.15 \times$ Age +4.305$)$. In addition, comparison of the clinical findings for the RIA-H and RIA-N groups found no difference in regard to the BMI, whereas the age was significantly $(\mathrm{p}=$ 0.0158) lower in the RIA-H group (Table 2).

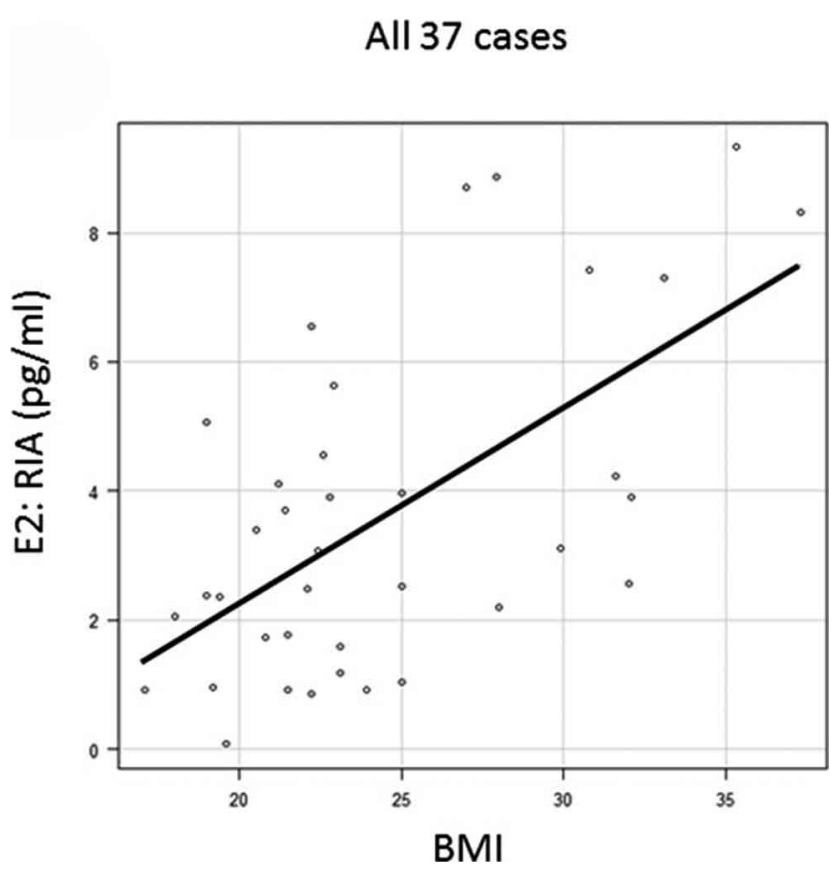

Figure $3 \mathrm{a}$ : Correlation between the E2 values by high-sensitivity RIA and the BMI

Patients with a high BMI had a significantly elevated E2 level, and the correlation was significant $(r=0.608, p=0.0007)$.

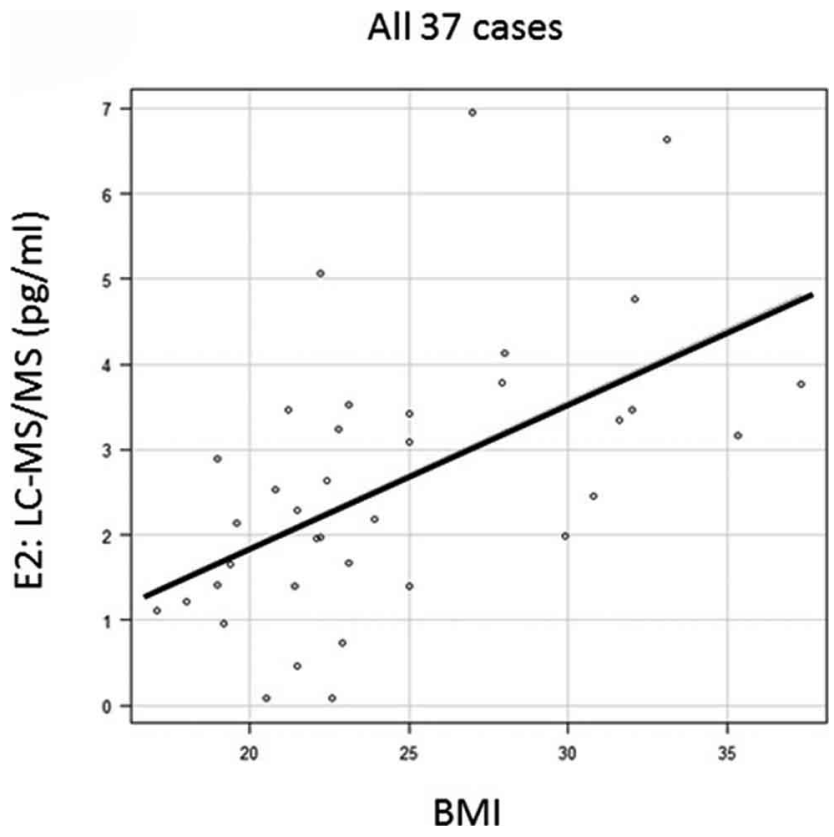

Figure $3 \mathrm{~b}$ : Correlation between the E2 values by LC-MS/MS and the BMI

Patients with a high BMI had a significantly elevated E2 level, and the correlation was significant $(r=0.554, p=0.000372)$.

\section{DISCUSSION}

AI drugs are the treatment of choice as endocrine therapy for hormone receptor-positive postmenopausal female breast cancer patients $(1,2)$. However, in some patients the serum E2 level does not decrease in response to AI therapy, making it necessary to monitor 
Table 2. Comparison of the clinical findings for RIA-N and RIA-H groups

\begin{tabular}{cccccccc}
\hline & All & & RIA-N & & RIA-H & P* \\
\hline Cases & 37 & & 28 & & 9 & & \\
& mean & SD & mean & SD & mean & SD & \\
Age & 51.6 & 6.6 & 53.3 & 5.9 & 46.7 & 6.4 & 0.00747 \\
Age of menarche & 12.3 & 1.2 & 12.3 & 1.2 & 12.3 & 1.3 & 0.98 \\
BMI** & 24.5 & 5.2 & 23.8 & 4.5 & 26.5 & 6.6 & 0.179 \\
Number of birth & 1.9 & 0.7 & 2 & 0.5 & 1.6 & 0.9 & 0.077 \\
\hline
\end{tabular}

*Welch's test ; **Body mass index

the serum E2 level in the early period of AI administration (5-8). Moreover, after AI drugs are administered, aromatase gene CYP19 polymorphism is involved in the serum level of E2 as well as manifestation of adverse reactions caused by the drugs (11-15). On the other hand, the serum E2 level is very low in postmenopausal women and below the limit of quantitation by the usual clinical assay methods, making it impossible to monitor. Accordingly, we carried out the present study aimed at elucidating the usefulness of highsensitivity RIA-which is the most sensitive assay method covered by insurance for use in ordinary clinics in Japan-in measuring the serum E2 level.

This study was conducted in female breast cancer patients for whom it was necessary to hormonologically confirm the menopausal status since amenorrhea had been caused by prior administration of anticancer drugs, etc. For that reason, the patient cohort was younger than patients who are naturally menopausal.

Our study found a weak but statistically significant positive correlation between the serum E2 data generated with the highsensitivity RIA and LC-MS/MS methods. Accordingly, we showed that the high-sensitivity RIA system used in this study can generally be used to monitor the serum E2 level in female postmenopausal breast cancer patients. We surmised that the reason that the correlation between the high-sensitivity RIA and LC-MS/MS assay results was weak overall was that there was a patient subgroup that showed higher E2 levels with high-sensitivity RIA compared with LC-MS/MS. For that reason, we stratified the patients into two groups, one consisting of patients whose E2 level by highsensitivity RIA was higher than that by LC-MS/MS (RIA-H group) and another comprised of the remaining patients (RIA-N group). Analysis of the data for those two groups showed a significant and strong correlation between the assay methods. These results for our study population thus suggest that there is a subgroup of patients who show a high serum E2 level when tested by the highsensitivity RIA method.

We next investigated what factors impact on the serum E2 values determined with the high-sensitivity RIA method. It was reported that the serum E2 level is high in obese women (10), so we analyzed for a correlation between obesity and the serum E2 level. Our results also showed that the serum E2 level increased together with the BMI. When we performed multiple regression analysis using the age, age at menarche, number of births and BMI as explanatory variables and by using the AIC for variable selection, the BMI was the only significant variable selected in the case of LCMS/MS, while both the BMI and age were significant variables in the case of high-sensitivity RIA. Those results thus confirm that the serum E2 level is affected by obesity. However, comparison of the background factors between the RIA-H group and the RIA-N group found no difference in the BMI. Accordingly, we found that the higher E2 levels seen with high-sensitivity RIA are not related to the BMI.

The next factor we investigated for a correlation with the serum E2 level was age. Our patient cohort included many patients who were young in comparison with ordinary women who are naturally menopausal. In our present results, the serum E2 levels determined with the high-sensitivity RIA method tended to be higher in the younger patients. It is unclear exactly how patient age is related, but it may be that some sort of humoral factor(s) in younger women is impacting on the RIA method. Our study did not include any investigation of the patients' eating habits, but since Japanese consume soybean protein, perhaps the isoflavones contained in soy products influence the serum E2 level. Because the serum E2 level is very low in postmenopausal women, cross-reactions and non-specific reactions will occur with the high-sensitivity RIA method, reducing the credibility of the assay results. The existence of a patient group showing high serum E2 levels with the highsensitivity RIA method may be due to such reactions, and identification of the causes is to be desired.

In order to further elucidate the usefulness of high-sensitivity RIA for monitoring serum E2 in patients starting on AI therapy, it will be necessary to carry out studies employing a much larger patient cohort that includes naturally menopausal women and also analyzes for possible influences from lifestyle factors, including dietary habits and components.

\section{CONCLUSION}

Although this study was conducted in a patient cohort that was limited in number and included young postmenopausal women with breast cancer, it demonstrated that the serum E2 levels determined with a high-sensitivity RIA method generally correlate with the results of LC-MS/MS. High-sensitivity RIA is thus concluded to be a useful method for serum E2 monitoring in patients starting on AI therapy. However, the results also suggested that in some Japanese women high-sensitivity RIA overestimates the serum E2 level compared with LC-MS/MS.

\section{COMPETING INTERESTS}

The authors declare that they have no competing interests.

\section{ACKNOWLEDGMENTS}

MS initiated and co-wrote the paper with MM, MT and JH took part in the care of patients. II performed the statistical analyses. TY, MY, HT, AT and II helped in preparation of the manuscript. All authors read and approved the manuscript.

\section{REFERENCES}

1. Goldhirsch A, Wood WC, Gelber RD, Coates AS, Thúrlimann 
B, Senn HJ : 10th St. Gallen conference. Progress and promise : highlights of the international expert consensus on the primary therapy of early breast cancer 2007. Ann Oncol 18 : 1133-1144, 2007

2. Winer EP, Hudis C, Burstein HJ, Wolff AC, Pritchard KI, Ingle JN, Chlebowski RT, Gelber R, Edge SB, Gralow J, Cobleigh MA, Mamounas EP, Goldstein LJ, Whelan TJ, Powles TJ, Brynt J, Perkins C, Perotti J, Braun S, Langer AS, Browman GP, Somerfield MR: American Society of Clinical Oncology technology assessment on the use of aromatase inhibitors as adjuvant therapy for postmenopausal women with hormone receptor-positive breast cancer : status report 2004. J Clin Oncol 23 : 619-629, 2005

3. Geisler J, Haynes B, Anker G, Dowsett M, Lonning PE : Influence of letrozole and anastrozole on total body aromatization and plasma estrogen levels in postmenopausal breast cancer patients evaluated in a randomized, cross-over study. J Clin Oncol 20 : 751-757, 2002

4. Geisler J, Detre S, Berntsen H, Ottestad L, Lindtiorn B, Dowsett $\mathrm{M}$, Einstein Lonning P : Influence of neoadjuvant anastrozole (Arimidex) on intratumoral estrogen levels and proliferation markers in patients with locally advanced breast cancer. Clin Cancer Res $7: 1230-1236,2001$

5. Nagao T, Kira M, Takahashi M, Honda J, Hirose T, Tangoku A, Zembutsu H, Nakamura Y, Sasa M : Serum estradiol should be monitored not only during the peri-menopausal period but also the post-menopausal period at the time of aromatase inhibitor administration. World J Surg Oncol 12(7) : 88, 2009

6. Burstein HJ, Mayer E, Patridge AH, O'Kane H, Litsas G, Come SE, Hudis CA, Goldstein DF, Muss HB, Winter EP, Garber $\mathrm{JE}$ : Inadvertent use of aromatase inhibitors in patients with breast cancer with residual ovarian function : cases and lessons. Clin Breast Cancer 7 : 158-161, 2006

7. Hargis JB, Nakajima ST : Resumption of menses with initiation of letrozole after five years of amenorrhea on tamoxifen : caution needed when using tamoxifen followed by aromatase inhibitors. Cancer Invest 24 : 174-177, 2006

8. Kunovac Kallak T, Baumgart J, Stavreus Evers A, Sundström Poromaa I, Moby L, Kask K, Norjavaara E, Kushnir MM,
Bergquist J, Nilsson K: Higher than expected estradiol levels in aromatase inhibitor-treated, postmenopausal breast cancer patients. Climacteric 15(5) : 473-480, 2012

9. Murakami K, Yamamoto Y, Iwase H : Choice of endocrine therapies for perimenopausal women with breast cancer considering serum estradiol levels. Jpn J Breast Cancer 25(1) : $37-42,2010$

10. Diorio C, Lemieux J, Provencher L, Hogue JC, Vachon E : Aromatase inhibitors in obese breast cancer patients are not associated with increased plasma estradiol levels. Breast Cancer Res Treat 136(2) : 573-9, 2012

11. Mao JJ, Su HI, Feng R, Donelson ML, Aplenc R, Rebbeck TR, Stanczyk F, DeMichele A : Association of functional polymorphisms in CYP19A1 with aromatase inhibitor associated arthralgia in breast cancer survivors. Breast Cancer Res 13(1) : 2011

12. Straume AH, Knappskog S, Lønning PE : Effect of CYP19 rs6493497 and rs7176005 haplotype status on in vivo aromatase transcription, plasma and tissue estrogen levels in postmenopausal women. J Steroid Biochem Mol Biol 128(1-2) : 69-75, 2012

13. Ghimenti C, Mello-Grand M, Grosso E, Scatolini M, Regolo L, Zambelli A, Chiorino G : Regulation of aromatase expression in breast cancer treated with anastrozole neoadjuvant therapy. Exp Ther Med 5(3) : 902-906, 2013

14. Fontein DB, Houtsma D, Nortier JW, Baak-Pablo RF, Kranenbarg EM, van der Straaten TR, Putter H, Seynaeve C, Gelderblom H, van de Velde CJ, Guchelaar HJ : Germline variants in the CYP19A1 gene are related to specific adverse events in aromatase inhibitor users : a substudy of Dutch patients in the TEAM trial. Breast Cancer Res Treat 144(3) : 599-606, 2014

15. Wang L, Ellsworth KA, Moon I, Pelleymounter LL, Eckloff BW, Martin YN, Fridley BL, Jenkins GD, Batzler A, Suman VJ, Ravi S, Dixon JM, Miller WR, Wieben ED, Buzdar A, Weinshilboum RM, Ingle JN : Functional genetic polymorphisms in the aromatase gene CYP19 vary the response of breast cancer patients to neoadjuvant therapy with aromatase inhibitors. Cancer Res 70(1) : 319-28, 2010 